\title{
Oral cavity changes during and after cancer therapy
}

\author{
Mihaela Furnadzhieva ${ }^{1}$, Svetoslav Slavkov ${ }^{2}$, Daniel \\ Markov $^{2}$, Rossen Kolarov ${ }^{2}$, Assya Krasteva ${ }^{1}$.
}

1. Faculty of dental medicine, Department of oral and imaging diagnostic, Medical University, Sofia, Bulgaria;

2. University Specialized Hospital for Maxillofacial Surgery, Medical University -Sofia, Bulgaria;

\begin{abstract}
Eight million people worldwide die of cancer every year. Professionals expect that their number will grow to 12 million per year by 2030. Oral cancer is currently the sixth most common carcinoma worldwide and squamous-cell carcinoma accounts for more than $90 \%$ of all cases. Treatment choices increase with advances in medicine but at the same time complications during and after cancer therapy become more frequent. The aim of this paper is to describe the possible side effects of oral cancer treatment on the oral cavity (xerostomia, mucositis, ulcerations, radiation caries, osteoradionecrosis, secondary infections) and the ways they can be avoided and treated.
\end{abstract}

Keywords: Oral cancer, radiation caries, osteoradionecrosis, xerostomia, mucositis, ulceration, secondary infections, trismus, dentofacial anomalies;

\section{Background}

A variety of acute reactions can be observed in the oral cavity during and after radiation and chemotherapy, depending on radiation dose and the type and nature of anticancer medications. Late side effects can occur several months or even years after radiation. 
Table 1. Possible changes occurring in the oral cavity following cancer therapy $(1,2,3,4,5)$

\begin{tabular}{|l|c|c|}
\hline & radiation therapy & chemotherapy \\
\hline Secondary infections & $\mathbf{X}$ & $\mathbf{X}$ \\
\hline Damage to bone marrow & $\mathbf{X}$ & $\mathbf{X}$ \\
\hline Dentofacial Anomalies & $\mathbf{X}$ & $\mathbf{X}$ \\
\hline Direct damage to the central nervous system & $\mathbf{X}$ & \\
\hline Dysphagia & & $\mathbf{X}$ \\
\hline Gingival bleeding and submucosal haemorrhages & $\mathbf{X}$ & \\
\hline Hypersensitive teeth & $\mathbf{X}$ & $\mathbf{X}$ \\
\hline Mucositis & $\mathbf{X}$ & \\
\hline Muscular dystrophy & $\mathbf{X}$ & \\
\hline Osteoradionecrosis & $\mathbf{X}$ & \\
\hline Periodontal disease & $\mathbf{X}$ & \\
\hline Radiation caries & $\mathbf{X}$ & \\
\hline Taste alterations & $\mathbf{X}$ & \\
\hline Trismus & $\mathbf{X}$ & $\mathbf{X}$ \\
\hline Ulceration & $\mathbf{X}$ & $\mathbf{X}$ \\
\hline Xerostomia & & \\
\hline
\end{tabular}

Table 2. Effects of radiation on mouth tissues $(6,7,8,9)$

\begin{tabular}{|l|l|}
\hline Mucosa & $\begin{array}{l}\text { atrophy, mucositis, vascular change, obliteration / stenosis of blood vessels, } \\
\text { reduced blood flow }\end{array}$ \\
\hline Muscles & fibrosis, vascular change \\
\hline Bones & decrease in osteocyte and osteoblast numbers, reduced blood flow \\
\hline Salivary glands & atrophy, fibrosis, vascular change \\
\hline Teeth & necrosis, reduced blood flow \\
\hline
\end{tabular}

\section{Xerostomia:}

Xerostomia is dryness in the mouth that may result from a direct or indirect salivary gland disorder occurring during or after cancer therapy. It presents as a partial, moderate or severe salivary deficiency and, occasionally, as a complete lack of salivation.

Unstimulated salivation rates lower than $0.1 \mathrm{ml} / \mathrm{min}$ measured for 5 to 10 minutes or stimulated salivation rates below $0.7 \mathrm{ml} / \mathrm{min}$ measured for 5 minutes suggest reduced salivary flow $(1,2,3,4,5)$.

Table 3. Amount of stimulated and unstimulated saliva

\begin{tabular}{|l|l|l|}
\hline & Output of saliva at rest & \multicolumn{1}{c|}{ Output of saliva after stimulation } \\
\hline Normal amount & $0,3-0,4 \mathrm{ml} / \mathrm{min}$ & $1-3 \mathrm{ml} / \mathrm{min}$ \\
\hline Low amount & $<0,1 \mathrm{ml} / \mathrm{min}$ & $<0,7 \mathrm{ml} / \mathrm{min}$ \\
\hline
\end{tabular}




\section{Effect of radiation therapy on salivary flow}

Saliva is essential to the quality of life since it helps humidify and soften food before swallowing and has a protective effect on oral mucosa and teeth. Salivary dysfunction results in changed sense of taste, foul breath, and may impede speech, swallowing, and eating. Moreover, oral dryness may involve change in oral $\mathrm{pH}$ levels which contributes to oral infections, tooth decay, and osteoradionecrosis (bone necrosis that does not recover over 3 to 6 months). Patients report xerostomia as a major factor deteriorating their quality of life (10).

Two weeks from the beginning of radiation therapy, saliva becomes more viscous or is nearly lacking. Its buffering capacity becomes impaired and the protective functions of the biological environment of the oral cavity are affected. As a result, $\mathrm{pH}$ level drops, leading to alterations in oral microflora. The ratio of cariogenic microorganisms such as Streptococcus mutans, Actinomyces, and Lactobacillus increases $(11,12)$.

First changes occur after radiation doses of $10 \mathrm{~Gy}$. Irreversible damage of salivary glands is associated with doses higher than 30 Gy. $(11,12)$.

Table 4. Effect of radiation therapy on salivary flow

destruction of the parenchyma: quantitative and qualitative change in the saliva

- increasing the viscosity

- saliva pH decreases

- reducing IG-A Img

- reducing the buffering capacity

- change of electrolytes

First changes occur at 10-Gy radiation.

Irreversible damage of the salivary glands is observed at 30-Gy radiation.

Table 5. Key factor in assessing glandular hypofunction include:

- radiation dose ;

- volume of glandular tissue exposed to radiations;

- type of radiation ;

- location of radiation fields;

- type of tissues exposed to radiation;

- patient's preparation for radiation therapy.

Patients are evaluated using the Overall Xerostomia Index to determine the extent of impairment of saliva flow. 
Table 6. OXI: Overall Xerostomia Index

\begin{tabular}{|l|l|}
\hline How severe is your dry & 1=normal \\
mouth problem? & 2= more dryness with respect to situation before radiation therapy \\
& 3= much more dryness with respect to situation before radiation therapy \\
& 1= persistant dryness \\
\hline Do you need to sip & = no \\
water to facilitate & 2= sometimes, depending on the quality of food \\
swallowing when & 3= more often than before \\
eating? & 4= with every bite of food \\
\hline Do you need to sip & 1= never \\
water to facilitate & 2= sometimes \\
speech? & 3= frequently \\
& 5= always \\
& 5= frequently have to interrupt speaking to take a sip of water \\
\hline
\end{tabular}

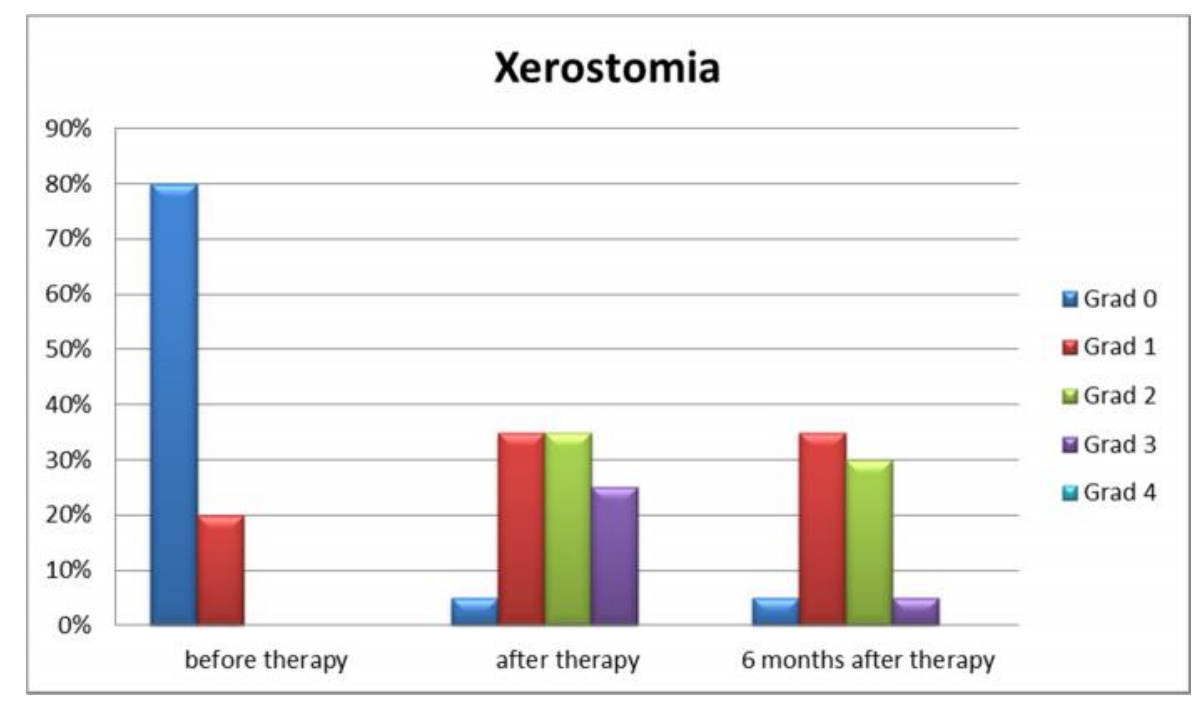

Figure 1. Xerostomia incidence before, after, and six months following radiation therapy (13).

A survey is conducted on 32 patients with head and neck squamous-cell carcinoma. Data are recorded before and 3 to 6 months after a six-week radiation therapy in doses of 55.8 to 72 Gy (14).

\section{Effects of chemotherapy}

Medication-induced xerostomia is also a frequent occurrence. There are more than 500 pharmaceutical products that may cause xerostomia, thus called xerogenic drugs. Medications have toxic effects causing sialadenosis and inducing salivary dysfunction as a result of metabolic and secretory damage of salivary gland parenchyma. Anticancer chemotherapeutic agents (e.g. doxorubicin) are considered to be directly 
responsible of salivary hypofunction. The xerogenic potential of drugs depends on dosage and duration of intake. High doses and long-term medication may result in salivary gland destruction $(11,12,15)$.

Residual salivary function can be stimulated by increased liquid intake and the use of chewing gum, sugar-free candy, and sialagogues (lemon, paraffin, etc.).

Another possibility is to administer substances that increase the saliva flow rate (pilocarpine, bethanechol, amifostine, etc.). Prior to prescribing such agents, it is crucial to measure stimulated saliva flow. If saliva levels are low at rest or after stimulation, the agent is unlikely to be effective. Pilocarpine, a parasympathomimetic drug, acts on the cholinergic receptors of the salivary gland cells: doses of 5 to 10 $\mathrm{mg}$ three times per day significantly increase salivary secretion.

Bethanechol (dosed 75-200 mg per day) belongs to the group of choline esters. It stimulates the parasympathetic nervous system and has a proven positive effect on the salivary glands by stimulating secretion without causing gastrointestinal disorders.

Amifostine for intravenous or intramuscular administration prior to radiation therapy is another drug approved by the Food and Drug Administration (FDA) for the prevention of salivary gland dysfunction and may reduce the severity of oral mucositis. Salivary gland stimulation during radiation therapy is a proven method of reducing salivary damage. Patients with high saliva flow before and in the beginning of radiation therapy present with a higher residual saliva flow. Preventive administration of pilocarpine (5 mg four times per day) in patients who will receive radiation therapy is thought to help preserve, at least partially, the parotid function. However, this effect has not been proven for submandibular and sublingual salivary glands (16).

Hyperbaric oxygen therapy (HBOT) is a new treatment method involving the inhalation of pure oxygen in a pressurized room for different periods and at different intervals. Increased oxygen concentration in the tissues leads to neovascularization of damaged tissue, increases fibroblast activity and collagen synthesis, and mobilizes stem cells. The effect of HBOT is present for years after completion of radiation therapy. The method is still under research (17).

High doses of radiation therapy lead to complete damage of the salivary function. These patients are recommended to avoid agents causing mucosal dryness such as tobacco, large amounts of caffeine, strong alcohol drinks, and spicy food. In such cases, the use of special products, such as artificial saliva in the form of gels, sprays, or mouth rinses (XEROSTOM, Dry Mouth Gel), is required to keep the oral mucosa moist. Another appropriate choice is Axerosta. It contains a mixture of emulsified lipids and softeners that form a film on the mucosa and keep it well moisturized. Its vitamin A and E contents help relieve the symptoms of mucosal dryness and have a soothing effect.

\section{Mucositis}

Mucositis is inflammation of oral mucosa resulting from immediate cytotoxic effect of radiation therapy or chemotherapy on the fast-dividing cells of oral epithelium. It develops mostly on non-keratinized mucosa in areas near metal fillings and prostheses. Mucositis starts with an inflammatory phase, followed by an ulcerative / bacteriological phase. Ulceration prompts the development of oral dysbiosis - Streptococcus mutans, Lactobacilli, Candida, and Gram-negative bacteria, leading to additional infections in the oral cavity and worsening of mucositis (16). 
More than 40 percent of all patients receiving cancer therapy suffer from mucositis. Most often, it develops between day 7 and day 14 after chemotherapy and after 14 days of radiation therapy in doses of 200 Gy per week $(1,2,3,4,5,6)$.

The first signs of oral mucositis include white coating of the oral mucosa due to epithelial hyperplasia/hypertrophy and intraepithelial oedema (mimicking burn-like lesions of the oral mucosa). Additional symptoms are: severe pain, loss of taste, dysphagia, and increased risk of other oral infections. Mucositis affects the quality of life and patients may need hospitalization.

Pain resulting from oropharyngeal mucositis often requires the administration of opioid analgesics. The risk of developing oral mucositis increases with poor oral hygiene, tobacco use, and salivary hypofunction (16).

Table 7. Oral mucositis - staging (WHO: World Health Organization)

\begin{tabular}{|l|l|}
\hline Grade I: & Painless mucosal erosion, erythema \\
\hline Grade II: & Moderately painful erythema, oedema, mucosal erosion, solid food intake possible \\
\hline Grade III: & Very painful erythema, intact basal membrane, liquid food intake possible \\
\hline Grade IV: & Ulceration, enteral/parenteral nutrition \\
\hline
\end{tabular}

Mucositis patients are recommended to avoid agents causing mucosal dryness such as tobacco, large amounts of caffeine, strong alcohol drinks, and spicy food. Mouth rinsing with salty water, baking soda, and chlorhexidine, a diet rich in salt and proteins, and the use of local analgesics (lidocaine $0.5 \%$ ) and/or antihistamine drugs and oral lubricants help achieve relief of symptoms (1).

Gelclair is a concentrated mouth gel for simultaneous treatment of oral mucositis and stomatitis. It acts by forming a protective film on exposed nerve endings in the damaged tissue, which results in pain relief. Ingredients include purified water, polyvinylpyrrolidone (PVP), sodium hyaluronate, glycyrrhetinic acid, maltodextrin, propylene glycol, PEG-40 hydrogenated castor oil, potassium sorbate, sodium benzoate, hydroxycellulose, benzalkonium chloride, EDTA, saccharine, and flavouring agents (18).

A novel treatment method consists of using CAM2028, a bioadhesive agent forming a lipid barrier to protect damaged tissue following radiation therapy. The application of CAM2028 has been proven to result in immediate and significant pain relief and at least $40 \%$ decrease in pain intensity (19).

Of non-drug treatment agents, curcuma and honey have been found to help relieve cancer-related mucositis (20).

\section{Ulcerations}

Oral cavity ulceration are very common in patients receiving antimetabolite and cytotoxic antibiotic treatment. Ulcerations typically appear shortly after the beginning of chemotherapy and may subside a few weeks after the end of treatment. Ulcerations cross the basement membrane. Although painful, they usually disappear within 2-3 weeks after cytotoxic therapy has been discontinued. 
Oral cavity ulceration are most common in treatment with methotrexate, 5-fluorouracil, doxorubicin, melphalan, mercaptopurine or bleomycin. Ulcers are often the first sign of methotrexate toxicity and may occur within 24 hours after a single dose.

A survey is conducted in 58 patients receiving chemotherapy. Examination of the oral cavity reveals various signs of inflammation of soft tissues of the mouth, such as ulcers, erythema, and erosion in $59 \%$ of all subject while $6 \%$ report strong pain causing difficulty swallowing and as much as $70 \%$ suffer mouth dryness, impaired taste, nausea, and vomiting (21).

\section{Table 8. Oral ulcerations and oncotherapy}

\begin{tabular}{|l|l|}
\hline & Number of patients with ulceration \\
\hline $\begin{array}{l}\text { Methotrexate } \\
\text { 5-Fluorouracil }\end{array}$ & $>50 \%$ \\
\hline Doxorubicin & $30 \%$ \\
\hline Mercaptopurine & $25 \%$ \\
\hline
\end{tabular}

\section{Secondary infections and oral candidiasis}

Patients having undergone chemotherapy and radiation therapy become susceptible to fungal, viral or bacterial infections. The main underlying cause is reduced salivary secretion and related negative effects. Candida albicans is, more than any other candida species, the most common cause of oral candidiasis. Candidiasis is a particularly frequent incidence in these patients also due to their immunosuppressed status following cancer treatment, severe leukopenia and antibiotic intake. Candidiasis may cause discomfort and alteration of taste. Since oral candidiasis does not lead to systemic infection unless the patient is immunocompromised, it can be treated with topical antifungal medications (16).

Oral carcinoma patients may also develop herpes infection, often presenting as chronic erosions and ulcers in the oral cavity.

Gram-positive bacterial (staphylococcal) infections are rarer since patients usually receive antibiotics as preventive treatment. However, hospitalized patients on cytotoxic chemotherapy may develop Gramnegative infections of the oral cavity (Pseudomonas, Enterobacter). Recovery from viral and bacterial infections is more problematic in immunosuppressed patients with white blood counts lower than 2000 cells/mm3 $(1,2,3,4,5)$.

\section{Taste alterations and Hypogeusia}

Alterations of taste and smell are a frequent occurrence after radiation therapy. The sense of taste may be affected directly due to the damage of the taste receptors or indirectly as a result from xerostomia or secondary infection. Hypogeusia (reduced sense of taste) is often present two weeks from the beginning of radiation therapy. Recovery of taste function is possible between month 2 and month 4 after completion of therapy. However, taste loss is permanent after radiation doses higher than 60 Gy $(6,11,12)$.

The intake of zinc (zinc sulphate in doses of $220 \mathrm{mg}$ twice a day) can be useful in patients with taste disorders (16). 


\section{Gingival bleeding and submucosal haemorrhages}

Cancer patients on radiation therapy or high-dose chemotherapy are susceptible to thrombocytopenia. Minor trauma resulting from biting or hygiene care procedures cause submucosal haemorrhage, gingival bleeding, and petechiae. Patients are recommended to maintain proper oral hygiene using antimicrobial rinses (often chlorhexidine preparations) and a softer toothbrush. Flossing and the use of toothpicks and interdental brushes are not recommended (1).

\section{Radiation caries}

Oral mucositis, bacterial and viral infections, mouth dryness, and taste loss during radiation therapy require often a special diet (softer food, rich in protein and carbohydrates). This, in addition to other factors such as decreased salivary secretion, reduced remineralizing potential, and loss of buffering capacity resulting in lower $\mathrm{pH}$, leads in turn to changes in bacterial flora and subsequent dental decay (22). As for radiation therapy, it affects directly dental structures by destroying tooth enamel, dentin, and pulp. Cancer therapy increases tooth sensibility, making oral hygiene more difficult. The above factors are as a whole the underlying cause of radiation caries.

Radiation caries affects most of all the cutting edge of the incisors, the tubercles of the canines, molars and premolars. Enamel surfaces may become involved at the level of the enamel - dentine junction $(6,11,12)$.

Radiation caries occurs between month 2 and month 10 after radiation therapy. Change in enamel starts 6 months after treatment. Even intact dentition may be affected within a year after therapy $(6,11,12)$.

Table 9. Radiation caries - staging

\begin{tabular}{|c|l|l|}
\hline Grade & \multicolumn{1}{|c|}{ Clinical characteristics } & \multicolumn{1}{c|}{ Procedure } \\
\hline $\mathbf{0}$ & N.A.D. & $\begin{array}{l}\text { Prevention } \\
\text { No treatment required }\end{array}$ \\
\hline \multirow{2}{*}{$\mathbf{1}$} & $\begin{array}{l}\text { Large chalk-like spots, } \\
\text { Loss of enamel brightness and } \\
\text { transparency, } \\
\text { Initial cervical caries }\end{array}$ & $\begin{array}{l}\text { - Improvement of oral hygiene } \\
- \text { Fluoride application } \\
\text { - Preventive crown placement } \\
\text { - Fillings }\end{array}$ \\
\hline $\mathbf{2}$ & Caries in enamel & $\begin{array}{l}\text { - Crowns } \\
\text { - Open pulp chamber - extraction }\end{array}$ \\
\hline $\mathbf{3}$ & $\begin{array}{l}\text { Subtotal or total loss of enamel } \\
\text { Beginning of dentin destruction }\end{array}$ & $\begin{array}{l}\text { Comprehensive surgical and restorative } \\
\text { treatment }\end{array}$ \\
\hline $\mathbf{4}$ & $\begin{array}{l}\text { Subtotal or total loss of tooth crown, } \\
\text { possible initial osteoradionecrosis }\end{array}$ & Surgical extraction \\
\hline
\end{tabular}

Maintaining a strict oral hygiene is crucial in cancer treated patients. The use of products stimulating residual salivary function and moistening the oral mucosa is strongly recommended. Tooth remineralization may be enhanced with supplemental fluoride in the form of fluoride gel applied with a mouth tray. In the beginning of the therapy, patients have to wear the tray 20 minutes per day. Later, this 
time is reduced to 5 minutes. Fluoride prevention of dental decay should be carried out for a long period of time $(11,12)$. The use of antimicrobial products with powerful effect against cariogenic bacteria such as Streptococcus mutans and Lactobacillus is of great value. An example of such product is chlorhexidine in the form of gel or mouth rinse (16).

In S3-LEITLINIE, German scholars recommend the use of a protective tray during irradiation to prevent radiation caries and reduce the destructive effect of radiation therapy on tooth enamel, dentine, and pulp (23).

\section{Osteoradionecrosis}

Mucosal or skin damage in radiogenic bone necrosis lasting for more than $\mathbf{3}$ months.

Radiation therapy triggers endarteritis which causes a decreased blood supply to the bones involving vascular hypoglycaemia and hypoxia. Therefore, trauma resulting from surgery, periodontal disease or pressure from ill-fitting dentures may cause necrosis. Typical symptoms include also discomfort or tenderness, pain, difficulty in opening the mouth, secondary infections, and pathological fractures. Over the last two decades, the risk of osteoradionecrosis has been assessed at 2.6 to $15 \%$, with the mandible being usually more affected $(11,12,16,24)$.

\section{Table 10. Osteoradionecrosis - staging (Schwartz)}

\begin{tabular}{|l|l|}
\hline Stage I: & Slight localised superficial bone resorption and exposure \\
\hline Stage II: & Major bone resorption and exposure \\
\hline Stage III: & Very deep bone damage, fistula, pathological fracture \\
\hline
\end{tabular}




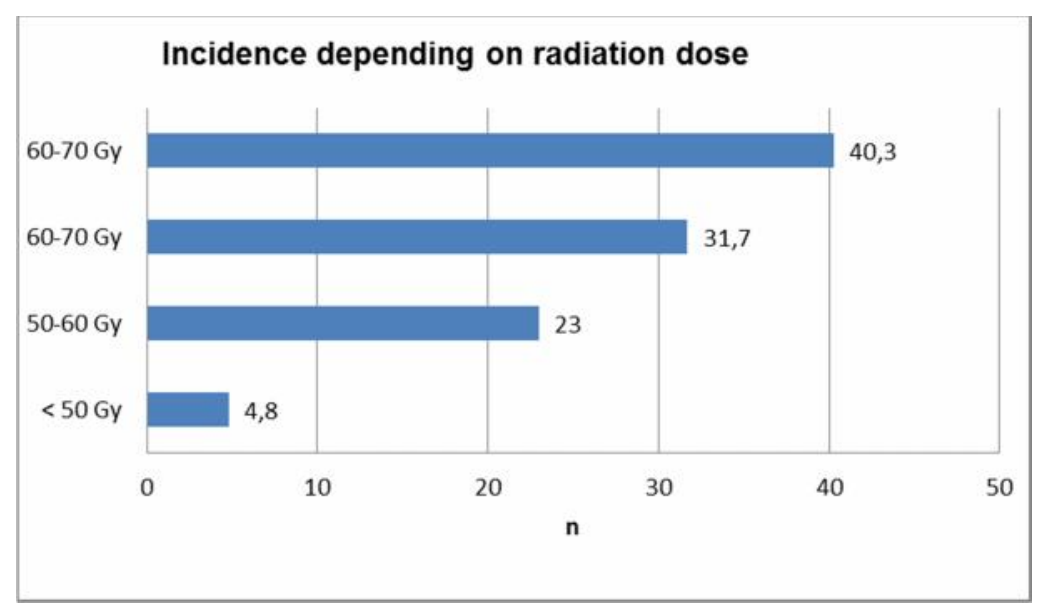

Figure 2. (25)

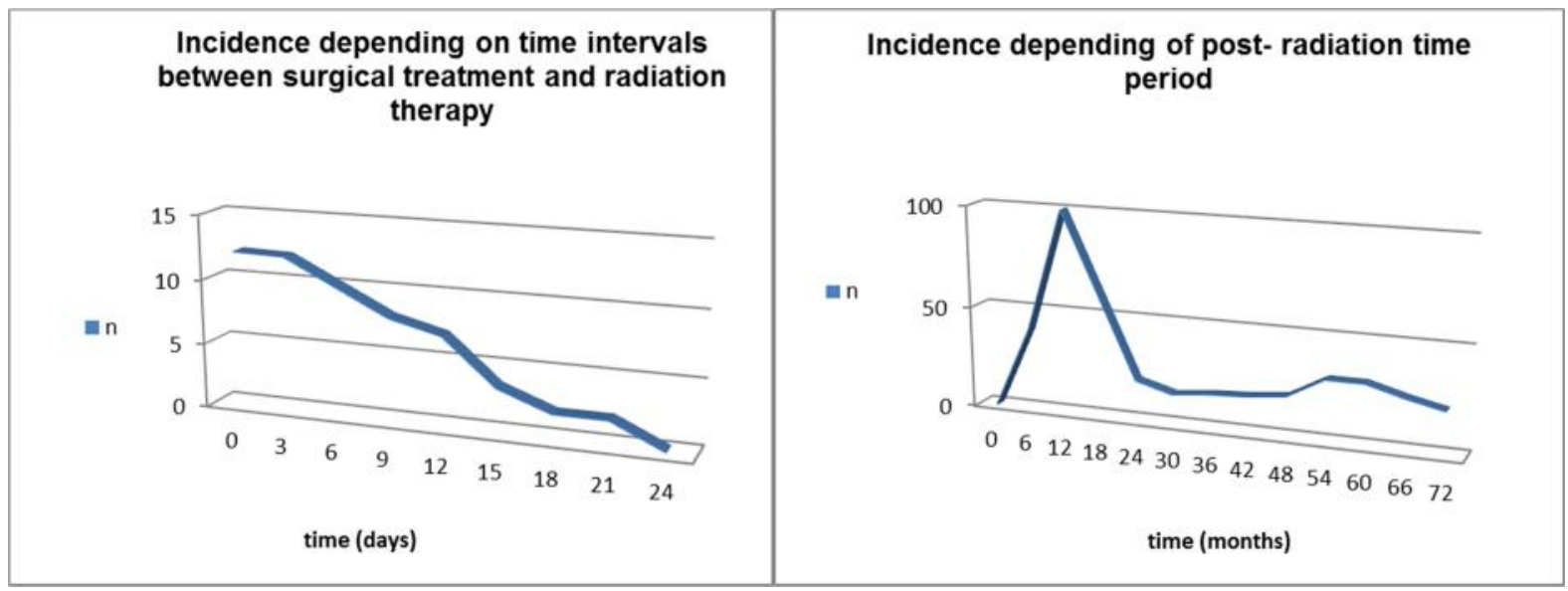

Figure 3 and 4. (25)

Preventive measures for improvement of oral hygiene taken by both doctor and patient before, during, and after cancer therapy and respecting the rules and indications for tooth extraction are crucial in the prevention of osteoradionecrosis (26). 
Table 11. Oral care before radiation therapy

\begin{tabular}{|l|}
\hline \multicolumn{1}{|c|}{ Oral care before radiation therapy } \\
\hline Comprehensive examination, evaluation of dental and periodontal status, X-ray diagnosis \\
\hline Patient education about possible side effects of radiation therapy \\
\hline Developing treatment plan \\
\hline Thorough atraumatic dental scaling \\
\hline Instructing the patient to improve dental hygiene \\
\hline Evaluating the patient's motivation \\
\hline Local fluoridation \\
- Making a custom-fitted mouth tray \\
- Fluoridation 5 min per day \\
- Alternative : $0,05 \%$ NaF solution 3 times per day \\
\hline Surgical removal of sharp bone edges and exostoses until 2 weeks prior to fluoride therapy \\
\hline Removal of orthodontic devices \\
\hline Making a protective tray \\
\hline Applying pilocarpine three times daily \\
\hline Advising the patient on follow-up \\
\hline
\end{tabular}

Table 12. Oral care during radiation therapy in patients with head and neck cancer

Weekly follow-up

Maintain oral hygiene during therapy

In the beginning or treatment patients should wear a fluoride tray 20 minutes per day then 5 minutes per day

Partial and total dentures should be used only when having meals

Placing new dental constructions during and immediately after therapy is excluded as a treatment option

Using a spacer

Surgery is excluded as a treatment option during radiation therapy 
Table 13. Oral care after radiation therapy in patients with head and neck cancer

\section{Control of oral hygiene and supplemental fluoride}

1. The patient is advised to use a fluoridation tray for 5 minutes per day ; In case of fluoride intolerance the use of a $0.05 \% \mathrm{NaF}$ solution is recommended

2. Mouth rinsing with $0.06 \%$ chlorhexidine is recommended to reduce plaque

\section{Prosthetic rehabilitation}

1. 6 to 12 after completion of radiation therapy

2. Avoiding pressure on the gingiva and mucosa

3. Tooth and/or implant-supported dental restorations are recommended

Surgery

1. If possible, not before 1 year after completion of therapy

2. Antibiotic intake before each visit

- Unacid (Ampicillin and Sulbactam), $\times 2$ tablets in the morning and in the evening , 3 days before and 10 days after surgery

3. Tooth extraction should be performed with minimal trauma, without osteotomy or curettage 4. Post-extraction wound should be sutured with well adapted flaps, without exposed bone structures and ischemic fields

5. Removal of sharp bone edges and exostoses

Table 14. Indications and rules for tooth extraction in patients before head and neck radiation therapy

\begin{tabular}{|l|}
\hline \multicolumn{1}{|c|}{ Indications } \\
\hline Teeth with poor or hopeless prognosis \\
Deep tooth decay \\
\hline Chronic / aggressive periodontitis \\
Bone pocket depth of $3.5 \mathrm{~mm}$ or more \\
Stage 3 and 4 PSI \\
\hline Apical periodontitis \\
\hline Impacted/semi-impacted teeth \\
\hline Healthy teeth on the background of a poor oral hygiene \\
\hline Healthy teeth on the background of a poor patient motivation \\
\hline \multicolumn{1}{|c|}{ Rules } \\
\hline Extractions with minimal trauma \\
\hline Surgical treatment should be completed 14 days prior to radiation therapy \\
\hline $\begin{array}{l}\text { Post-extraction wound should be sutured with well adapted flaps, without exposed bone structures and } \\
\text { ischemic fields }\end{array}$ \\
\hline Extraction of symptomatic devitalised teeth should be performed 14 days prior to radiation therapy \\
\hline
\end{tabular}

Early stage osteoradionecrosis can be treated with antibiotics and locally by removing contaminated necrotic tissue and disinfection. The aim of treatment is to allow the formation of new connective tissue 
and stimulate angiogenesis. Patients in advanced stages of the disease are treated by decortication, continuous resection with or without tissue graft, and microvascularization.

It is important to note that oncotherapy of metastatic bone carcinoma of breast, lung, kidney, and prostate cancer may also give rise to osteonecrosis. This type of disease is termed Bisphosphonate-Related Osteonecrosis of the Jaw (BRONJ) and it is a frequent side effect of bisphosphonate cancer therapy. Patients suffering from BRONJ do not receive radiation therapy. They present with exposed, non-vital bone, which does not recover for more than 8 weeks after current or past bisphosphonate therapy. These medications have an anti-osteoclast effect and are administered to slow down bone loss and preserve existing bone. To prevent osteonecrosis, surgical periodontal and endodontic treatments should be completed before bisphosphonate therapy. Similar interventions may be performed at least 14 days after bisphosphonate intake or preventive antibiotic treatment. The patient should not use the drug for another 14 days after the intervention (27).

\section{Dysphagia}

Each type of oral carcinoma treatment (surgery, radiation therapy, and chemotherapy) may result in some degree of dysphagia due to progressive tissue fibrosis and reduced salivation, often complicated with oral mucositis and mucosal ulceration.

Patients complain of pain in the oral cavity, a sense of "irritation and foreign body in the throat", and difficulty in swallowing. Even soft food intake may cause discomfort.

Other symptoms include burning sensation when swallowing and accelerated heart rate as a result of the intake of hot beverages. The onset of the first symptoms of dysphagia comes as early as 2 to 3 weeks from the beginning of radiation therapy.

Severe impairment of swallowing subsists for years after treatment has been completed.

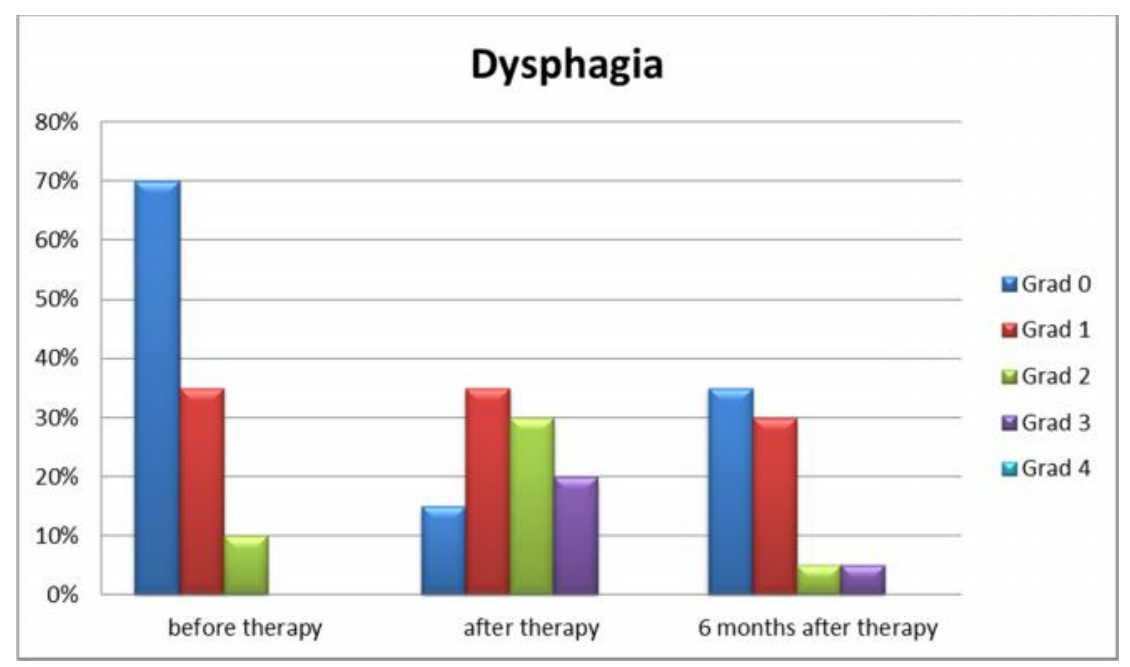

Figure 5. Dysphagia incidence before, after and 6 months after radiation therapy (13). 
A survey is conducted on 32 patients with head and neck squamous-cell carcinoma. Data are recorded before and 3 to 6 months after a six-week radiation therapy in doses of 55.8 to 72 Gy.

\section{sTable 15. Dysphagia - staging (Atkinson)}

\begin{tabular}{|l|l|}
\hline Grade 1: & Difficulty swallowing solid foods only \\
\hline Grade 2: & Need to have soft foods pureed \\
\hline Grade 3: & Liquid food needed \\
\hline Grade 4: & Liquid food intake becomes impossible \\
\hline
\end{tabular}

\section{Muscular dystrophy and Trismus}

A progressive endarteritis (an inflammation of the inner layer of the arteries) is observed in the affected tissues. The consequence is fibrosis of the muscles of mastication. Its onset comes 3 to 6 months after radiotherapy and may lead to permanent restriction of normal ability to open the mouth (trismus) $(6,11,12)$. Jaw-stretching exercises, micro current electrotherapy (MET), and medication (Pentoxiphylline) help reduce fibrosis and improve limited mandibular movements, which appears to be the appropriate approach in patients with this problem. (16).

\section{Table 16. Trismus - staging}

\begin{tabular}{|l|l|}
\hline Stage I: & opening degree subjectively reduced \\
\hline Stage II: & degree of opening up to $10 \mathrm{~mm}$ \\
\hline Stage III: & degree of opening $1 \mathrm{~mm}$ \\
\hline
\end{tabular}

Impairment of speech and mastication is a frequent symptom after partial of total maxillectomy. Restoring the physiology of swallowing, speech and mastication requires the placement of obturator prosthesis to improve function $(16,28)$.

\section{Dentofacial Anomalies}

Radiation therapy in children may affect growth of facial skeletal structures and the development of the dentomaxillary complex. Irradiation of developing teeth may cause hypoplasia, delayed or arrested development of teeth, unusual root form or abnormal calcification. Despite radiation therapy, teeth may grow and be preserved for years $(1,2)$.

The maturation of facial skeletal structures may also be affected by irradiation. The irradiation of growth centres in children may result in craniofacial malformations: micrognathia, retrognathia, and jaw changes and asymmetry (16).

Pain 
Pain is the major symptom accompanying surgery, radiation therapy, and chemotherapy in patients with head and neck carcinoma. It affects the patients' overall condition, increases anxiety and depression, causes sleep disorders, and deteriorates quality of life (QOL) (16).

\section{References}

1. Little J, Falace D, Miller Cr et al. Dental Management of the Medically Compromised Patient, sixth edition 2002, Mosby.

2. Sonis St, Fazio R, Fang L, Oral medicine secrets, 2003. Hanley \& Belfus.

3. Scully C, Oral and Maxillofacial Medicine; The basis of diagnosis and treatment, 2004. Wright.

4. Scully C, Cawson R. Medical Problems in Dentistry, Fourth edition 1998. Wright.

5. Mitchell D, Mitchell L, Oxford Handbook of Clinical Dentistry, fourth edition, 2005. Oxford.

6. Krastev Z, Kiselova A, Kolarov R. Oral medicine (In Bulgarian), 2009. Ivan SapundzhievEOOD

7. Fletcher C.D.M. The evolving classification of soft tissue tumors: an update based on the new WHO classification, 2006.

8. Kwon P H, Daniel M L. Clinicians Manual of Oral and Maxillofacial Surgery, 2001.

9. Mendenkall W.M, Werning J.W, Adult head and neck soft tissue sarcomas. Head Neck, 2005.

10. Pellegrino F, Groff E, Bastiani L, Fattori B, Sotti G. Assessment of radiation-induced xerostomia: validation of the Italian version of the xerostomia questionnaire in head and neck cancer patients. Support Care Cancer 2015; 23:4:925-932.

11. Schwenzer N, Ehrenfeld M, Dental, Oral and Maxillofacial Surgery: Surgical Basics (In German), fourth edition, 2008. Thieme.

12. Schwenzer N, Ehrenfeld M, Dental, Oral and Maxillofacial Surgery: Oral and maxillofacial surgery (In German), fourth edition, 2010. Thieme.

13. Universitätsklinikum Regensburg Klinik und Poliklinik für Strahlentherapie, 2007.

14. Schaefer Chr, Hipp M, Kutz J, Schneider P, Koelbl O, Xerostomia and quality of life before, during and 6 months after IMRT and 3D Konformationsstrahlentherapiq in squamous cell head and neck region, (In German), Regensburg. 
15. Dzhemileva, .; Xerostomia - etiology, clinical and therapeutic approaches, Acer, (In Bulgarian), 1998.

16. Burket L, Greenberg M, Glick M et al. Burket's Oral Medicine. PMPH-USA, 2008.

17. Fox NF, Xiao C, Sood AJ et al. Hyperbaric oxygen therapy for the treatment of radiationinduced xerostomia: a systematic review. Oral Surg Oral Med Oral Pathol Oral Radiol 2015; 120:1:22-28.

18. http://www.gelclair.com/

19. Hadjieva T, Cavallin-Ståhl E, Linden M, Tiberg F. Treatment of oral mucositis pain following radiation therapy for head-and-neck cancer using a bioadhesive barrier-forming lipid solution. Support Care Cancer 2014; 22:6:1557-1562.

20. Francis M, Williams S. Effectiveness of Indian Turmeric Powder with Honey as Complementary Therapy on Oral Mucositis: A Nursing Perspective among Cancer Patients in Mysore. Nurs J India 2014; 105:6:258-260.

21. Rahnama M, Madej-Czerwonka B, Jastrzębska-Jamrogiewicz I, Jamrogiewicz R, Analysis of the influence of parenteral cancer chemotherapy on the health condition of oral mucosa. Contemp Oncol 2015; 19:1:77-82.

22. Jensdottir T, Buchwald C, Nauntofte B, Hansen HS, Bardow A. Saliva in relation to dental erosion before and after radiotherapy. Acta Odontol Scand 2013; 71:3:1008-1013.

23. S3-LEITLINIE, Das Mundhöhlenkarzinom, Zm online, HEFT 23/2012 ZAHNMEDIZIN.

24. Frerich B, Osteoradionecrosis (In German), Department of Oral and Facial Plastic Surgery 2013, Rostock.

25. Brauckhoff G, Kocher T, Holtfreter B et al. Oral health, Robert Koch-Institut, Berlin 2009. Gesundheitsberichterstattung des Bundes.

26. Niewald M, Mang K, Barbie et al. Dental status, dental treatment procedures and radiotherapy as risk factors for infected osteoradionecrosis (IORN) in patients with oral cancer - a comparison of two 10 years' observation periods. Springerplus 2014; 3:263.

27. Vescovi P, Nammour S. Bisphosphonate-Related Osteonecrosis of the Jaw (BRONJ) therapy. A critical review. Minerva Stomatol 2010; 59(4):181-203, 204-213.

28. Zahariev Z, Hadzhieva T, National standards for radiotherapy of cancers of the oral cavity, (In Bulgarian), 2010. 


\section{Corresponding author:}

Assya Krasteva

Faculty of dental medicine, Department of oral and imaging diagnostic, Medical University, Sofia, Bulgaria

1 Georgi Sofiyski blvd., 1431 Sofia, Bulgaria

e-mail:asyakrasteva@abv.bg 